TITLE:

\title{
Numerical study of drying process and columnar fracture process in granule-water mixtures
}

$\operatorname{AUTHOR}(S)$ :

Nishimoto, A; Mizuguchi, T; Kitsunezaki, S

\section{CITATION:}

Nishimoto, A ... [et al]. Numerical study of drying process and columnar fracture process in granule-water mixtures. Physical Review E 2007, 76(1): 016102.

ISSUE DATE:

2007-07

URL:

http://hdl.handle.net/2433/49943

RIGHT:

Copyright 2007 American Physical Society 
PHYSICAL REVIEW E 76, 016102 (2007)

\title{
Numerical study of drying process and columnar fracture process in granule-water mixtures
}

\author{
Akihiro Nishimoto* \\ Department of Physics, Kyoto University, Kyoto 606-8502, Japan \\ Tsuyoshi Mizuguchi \\ Department of Mathematical Sciences, Osaka Prefecture University, Sakai 599-8531, Japan \\ So Kitsunezaki \\ Graduate School of Human Culture, Nara Women's University, Nara 630-8506, Japan
}

(Received 1 February 2007; published 3 July 2007)

\begin{abstract}
The formation of three-dimensional prismatic cracks in the drying process of starch-water mixtures is investigated numerically. We assume that the mixture is an elastic porous medium which possesses a stress field and a water content field. The evolution of both fields is represented by a spring network and a phenomenological model with the water potential, respectively. We find that the water content distribution has a propagating front which is not explained by a simple diffusion process. The prismatic structure of cracks driven by the water content field is observed. The depth dependence and the coarsening process of the columnar structure are also studied. The particle diameter dependence of the scale of the columns and the effect of the crack networks on the dynamics of the water content field are also discussed.
\end{abstract}

DOI: $10.1103 /$ PhysRevE.76.016102

PACS number(s): 62.20.Mk, 46.50.+a, 45.70.Qj, 47.56.+r

\section{INTRODUCTION}

Crack patterns are observed in everyday life [1]. Columnar joints in cooled lava, especially, are an intriguing phenomenon and has fascinated many people for centuries and has been studied by field work [2-5]. Theoretical studies have been carried out from the viewpoint of the analysis of the thermal field [6-8], the ordering of patterns $[9,10]$, and size selection of columns by the finite element method [11]. However, many questions remain open, such as, "Why are the prismatic structures with the polygonal cross sections formed?," "How are the size of columns and the distribution of polygon types determined?" The scale of columns is 0.1 $\sim 1 \mathrm{~m}$ in diameter, hence it is too difficult to study the fracture process of cooled lava experimentally.

Recently, patterns of cracks similar to columnar joints have been investigated in the drying process of starch-water mixtures [12-18]. Well-controlled experiments are possible due to the small scale of columns $(1 \sim 10 \mathrm{~mm}$ in diameter). In typical experiments of these studies, after the mixtures of starch and water are poured into a container, water evaporates from the sample surface. During the drying process there are three stages $[13,15,17,18]$. Stage 1: The water content decreases uniformly. Stage 2: Cracks are formed which extend to the bottom of the sample and a sudden change of water content occurs. These cracks have a uniform structure along the vertical direction and are referred to as the primary (type I) cracks $[13,17,19-21]$. In the case that the thickness of a sample is sufficiently large, the primary cracks appear only between the mixture and the side wall of the container. Stage 3: The water content decreases slowly and the water content distribution becomes nonuniform, i.e., it has a "front" $[17,18]$. The front propagates inward and the mixture

*nisimoto@ton.scphys.kyoto-u.ac.jp shrinks nonuniformly and another type of cracks, i.e., the secondary (type II) cracks, are formed. The secondary cracks show three-dimensional prismatic structure. The size of columns depends on the evaporation rate $[15,16]$ and it coarsens with the distance from the sample surface $[16,17]$. In this paper, we focus on the secondary cracks.

By a numerical model, Hayakawa [22] showed that a regular prismatic crack structure is formed by the steady sweep of external field (temperature) with fixed shape. Experimental results on drying starch, however, suggest that the shape of the external field (water content) changed temporally. In order to study the crack pattern of starch-water mixtures, the drying process and the fracture process should be taken into account three dimensionally. Combined numerical analysis of both processes, however, has not been performed yet. The drying process involving all stages, especially, has not been analyzed, mainly because water transport in porous media is a complicated process.

The nonuniform shrinkage due to the change of the external field causes directional propagation of cracks and the cracks become the boundary condition of the external field, simultaneously. Changes in the external field can be induced by the crack boundary conditions, as suggested by some studies [23-25]. This type of crack is referred to as a selfdriven crack. It has neither been discussed in detail nor demonstrated numerically whether this effect is important or not in the case of starch-water mixtures. In some studies on columnar joints $[6,7,9,26,27]$ and desiccation cracks $[17,28]$, the effect of cracks as the boundary condition is considered to play an important role. On the other hand, in other studies such as two-dimensional directional fracture of glass strips [29-31], columnar joints [8], and desiccation cracks $[12,13,18,32-34]$, this effect is hypothesized not to be important.

In this paper, our goal is a comprehensive description of the water content distribution and the three-dimensional realization of the crack formation process driven by the non- 


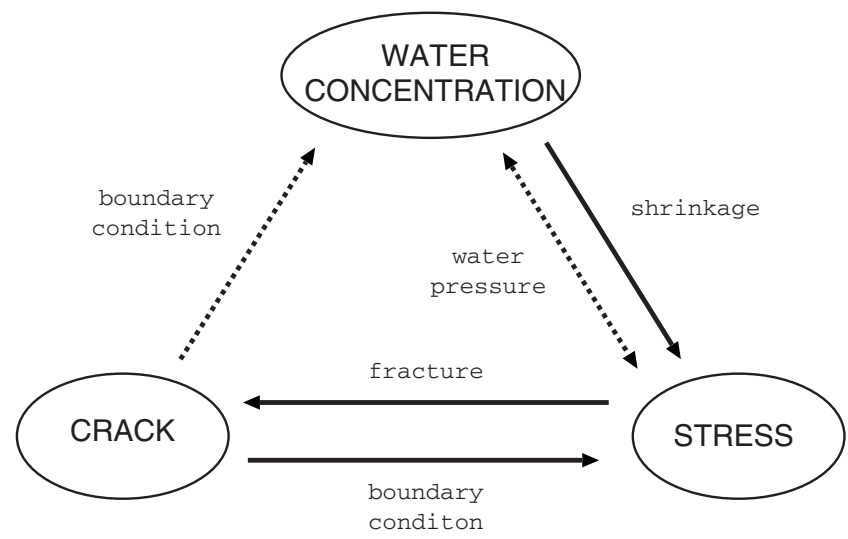

FIG. 1. Schematic diagram of the model. Three elements and interactions between them are drawn. The interactions indicated by solid arrows are taken into account in our model while the dashed ones are ignored.

uniform shrinkage during the drying process of starch-water mixtures. This paper is organized as follows. In Sec. II, details of the model are described. We assume that the mixture is an elastic porous medium which has a stress field and a water content field. The evolution of the former is represented by a spring network model and the latter is represented by a phenomenological model with the water potential. Here we suppose that cracks do not affect the evolution of the water content distribution. In Sec. III, numerical results are presented. A propagating front of water content distribution and the prismatic pattern of cracks driven by the water content field are obtained, which well capture those observed in the experiments. The particle diameter dependence of the scale of columns is suggested. In Sec. IV, the properties of the drying front and the validity of some assumptions used in our model are discussed. The effect of the crack networks on the dynamics of the water content field and the interaction between the stress field and the water content field are considered. Geological columnar joints and the characterization of the crack patterns are discussed. Finally, we conclude this paper with a summary in Sec. V.

\section{MODEL}

The drying fracture process consists of various factors. Here, as key factors, we focus on the following quantities and processes (Fig. 1): the change of water content by the evaporation and transportation of liquid water and vapor in porous media, the nonuniform volumetric contraction and the stress intensification, the formation of cracks, and the change of the boundary condition of the stress field.

Starch-water mixtures are elastic porous media composed of solid phase (granules), liquid phase (water), and gas phase (air and vapor). As the water content decreases by drying, the state of the mixture changes from "capillary fringe" (liquid is saturated under negative pressure) to "funicular" (liquid is unsaturated and contiguous), and finally to "pendular" (liquid is isolated) [35]. This drying process is mainly driven by the transportation of liquid water and vapor, and the phase change between them. In this paper, we adopt the water potential [36] as one of the key field variables.
For simplicity, we assume that the interactions between the crack network and the water content field, the influences of the water pressure on the stress field, and the influences of the stress field on the water potential, indicated by dashed lines in Fig. 1, are negligible. Our numerical simulations, however, exhibit good realizations of the experimental results as shown in the next section. The validity of these assumptions are discussed in Sec. IV.

The stress field and its boundary are represented by the network of springs. And the water content field and its evolution are represented by the equations for the water potential. The details of the model are stated in the following subsections, i.e., the stress field, the water content field using the water potential, and the interaction between them and their evolution.

\section{A. Stress field}

We construct a cubic lattice with nearest-neighbor (NN) and next-nearest-neighbor (NNN) interactions in order to calculate the stress field [22]. Each interaction is represented by a Hookean spring. The lattice constant is $a_{0}$ and the size of the system is $L_{x} \times L_{y} \times L_{z}$. The lattice point is expressed as $\alpha \equiv(X, Y, Z)$, where $0 \leqslant X \leqslant N_{x}, 0 \leqslant Y \leqslant N_{y}, 0 \leqslant Z \leqslant N_{z}, L_{x}$ $=N_{x} a_{0}, L_{y}=N_{y} a_{0}, L_{z}=N_{z} a_{0}$. The $z$ axis is chosen as the depth, the top (bottom) is set to $z=0\left(z=L_{z}\right)$.

Let the spring constants for the nearest-neighbor and nextnearest-neighbor interactions be $k_{1}$ and $k_{2}$, respectively. Then we obtain a spring network of the elastic tensor $C_{i j k l}$ with the components [22]

$$
\begin{gathered}
C_{i i i i}=\frac{k_{1}+2 k_{2}}{a_{0}}, \\
C_{i j i j}=C_{i j j i}=C_{i i j j}=\frac{k_{2}}{a_{0}} .
\end{gathered}
$$

We assume that the water content field changes very slowly and the stress field relaxes much faster than the water content field. So the stress field is assumed to be balanced at each moment under conditions induced by the water content field and the boundary condition at that time. We assume that each spring is broken at a certain critical force, i.e., if the force given on a spring exceeds a constant $F_{c}$, the spring constant is set to be zero irreversibly. Using a position vector $\mathbf{r}_{\alpha}$ of the lattice point $\alpha$, the force acting on the lattice point $\alpha$ is represented by

$$
\begin{aligned}
& \mathbf{F}_{\alpha}= \sum_{\beta \in \mathrm{NN}} K_{1}^{\alpha \beta} \frac{\mathbf{r}_{\alpha}-\mathbf{r}_{\beta}}{\left|\mathbf{r}_{\alpha}-\mathbf{r}_{\beta}\right|}\left(a_{\alpha \beta}-\left|\mathbf{r}_{\alpha}-\mathbf{r}_{\beta}\right|\right) \\
&+\sum_{\beta \in \mathrm{NNN}} K_{2}^{\alpha \beta} \frac{\mathbf{r}_{\alpha}-\mathbf{r}_{\beta}}{\left|\mathbf{r}_{\alpha}-\mathbf{r}_{\beta}\right|}\left(a_{\alpha \beta}-\left|\mathbf{r}_{\alpha}-\mathbf{r}_{\beta}\right|\right), \\
& K_{i}^{\alpha \beta}= \begin{cases}k_{i} & (\text { connected }, i=1,2), \\
0 & \text { (broken), }\end{cases}
\end{aligned}
$$

where $a_{\alpha \beta}$ is the natural length of the spring which connects lattice points $\alpha$ and $\beta$. The equilibrium condition of the 
spring network, $\mathbf{F}_{\alpha}=0$, is equivalent to the condition that the elastic energy

$$
E=\sum_{\alpha} E_{\alpha},
$$

where

$$
\begin{aligned}
E_{\alpha}= & \sum_{\beta \in \mathrm{NN}} \frac{1}{2} K_{1}^{\alpha \beta}\left(a_{\alpha \beta}-\left|\mathbf{r}_{\alpha}-\mathbf{r}_{\beta}\right|\right)^{2} \\
& +\sum_{\beta \in \mathrm{NNN}} \frac{1}{2} K_{2}^{\alpha \beta}\left(a_{\alpha \beta}-\left|\mathbf{r}_{\alpha}-\mathbf{r}_{\beta}\right|\right)^{2},
\end{aligned}
$$

is minimal under the given boundary condition and the given natural length of each spring. In our simulations, the free boundary condition is imposed. Because no friction acts on the boundary of the system, the primary cracks do not appear.

\section{B. Water content field}

The evolution of the water content in unsaturated porous media consists of various processes. Here, we use the Campbell's desiccation model [37] that includes the transportation of liquid water and vapor and the evaporation. By assuming that the temperature is constant and the speed of evaporation is slow, the heat of evaporation is ignored. The evolution equation is described by one variable because both the liquid water content and the density of vapor are determined from the water potential as described below.

The effect of the deformation of the spring network on the water content field is ignored and the evolution equation is solved on the undeformed lattice in the Cartesian coordinates system because the change of the total volume is smaller than that of water volume [13]. The change of the boundaries due to the formation of cracks is also ignored. Note that there are many field variables and constants in this model. In order to distinguish them all the field variables are expressed by the symbols with an argument of position $\mathbf{r}$ (or $z$ ) and time $t$.

The water potential is defined as the chemical potential per unit mass of water in the mixtures, compared to that of pure, free water $[35,37,38]$. The potential is generally negative for unsaturated mixtures. Although this potential is the sum of several components, some components are negligible. The effect of gravity is negligible because the size of a starch-water specimen in the experiments is on the order of $1 \mathrm{~cm}$. We carried out the experiment [39] to investigate the effect of gravity and confirmed that the columns normal to the free surface are formed even when the desiccated starchwater mixture in a container is laid on its side. We assume that there is no external pressure and the water in mixtures is pure, so we can ignore the overburden potential and the osmotic potential. Hence, the water potential is well represented by the matric potential $\psi_{m}(\mathbf{r}, t)$. The matric potential is defined as the amount of work per unit mass of water, required to transport an infinitesimal quantity of water from the mixture to a reference pool of water at the same elevation, pressure, and temperature. The capillary water between particles and the water on the particle surface contribute to the matric potential. Below we use the matric potential as the water potential, i.e., the chemical potential per unit mass of water in mixtures. The contribution of the capillary water to the potential is expressed as

$$
\frac{P_{\text {cap }}}{\rho_{L}},
$$

where $P_{\text {cap }}$ is the capillary pressure, expressed as $-2 \sigma / r_{\text {cap }}, \sigma$ is the surface tension of water and $r_{\text {cap }}$ is the inverse of the mean curvature of the capillary water in the mixture.

From the coexistence condition of the liquid phase and the gas phase, the relative humidity $h(\mathbf{r}, t)$ is related to the matric potential $\psi_{m}(\mathbf{r}, t)$ by the Kelvin equation

$$
h(\mathbf{r}, t)=\exp \left(\frac{M_{w}}{R T} \psi_{m}(\mathbf{r}, t)\right),
$$

where $M_{w}$ is the molecular weight of water, $R$ is the gas constant, and $T$ is the absolute temperature.

Let $\theta_{L}(\mathbf{r}, t)$ denote the volumetric water content. Assuming that $\theta_{L}(\mathbf{r}, t)=\theta_{S}$ in the saturated state $\left(\theta_{S}\right.$ corresponds to the porosity of the mixture), the volumetric gas content $\theta_{G}(\mathbf{r}, t)$ is given as

$$
\theta_{G}(\mathbf{r}, t)=\theta_{S}-\theta_{L}(\mathbf{r}, t) .
$$

During the drying process, $\theta_{L}(\mathbf{r}, t)$ decreases as $\theta_{G}(\mathbf{r}, t)$ increases. $\theta_{S}$ is assumed to be constant because we ignore the feedback from the stress field (fracture and deformation) to the water content field and the change of the pore volume due to the change of the water content.

Expressing the fluxes of liquid water and vapor as $J_{L}(\mathbf{r}, t)$ and $J_{V}(\mathbf{r}, t)$ and the evaporation rate per unit volume as $W_{\text {eva }}(\mathbf{r}, t)$, the transportation equation of liquid water and vapor is given by

$$
\begin{gathered}
\frac{\partial}{\partial t}\left[\rho_{L} \theta_{L}(\mathbf{r}, t)\right]=-\nabla \cdot J_{L}(\mathbf{r}, t)-W_{\mathrm{eva}}(\mathbf{r}, t), \\
\frac{\partial}{\partial t}\left[\rho_{V}(\mathbf{r}, t) \theta_{G}(\mathbf{r}, t)\right]=-\nabla \cdot J_{V}(\mathbf{r}, t)+W_{\mathrm{eva}}(\mathbf{r}, t),
\end{gathered}
$$

where $\rho_{L}$ is the density of liquid water and $\rho_{V}(\mathbf{r}, t)$ is the density of vapor. The incompressibility of water is assumed, i.e., $\rho_{L}$ is constant.

In unsaturated porous media, the flux of water is assumed to be proportional to the gradient of the water potential (Darcy's law) $[35,37,38]$

$$
J_{L}(\mathbf{r}, t)=-k_{L}(\mathbf{r}, t) \boldsymbol{\nabla} \psi_{m}(\mathbf{r}, t),
$$

where $k_{L}(\mathbf{r}, t)$ is the hydraulic conductivity. The properties of starch-water mixtures in the drying process, such as the dependences of the hydraulic conductivity $k_{L}(\mathbf{r}, t)$ and the water potential $\psi_{m}(\mathbf{r}, t)$ on the volumetric water content $\theta_{L}(\mathbf{r}, t)$, have not been investigated in detail $[40,41]$. Here, we suppose that the properties of starch-water mixtures are similar to those of soil-water systems and employ the relations confirmed by soil mechanics experiments $[35,37]$, 

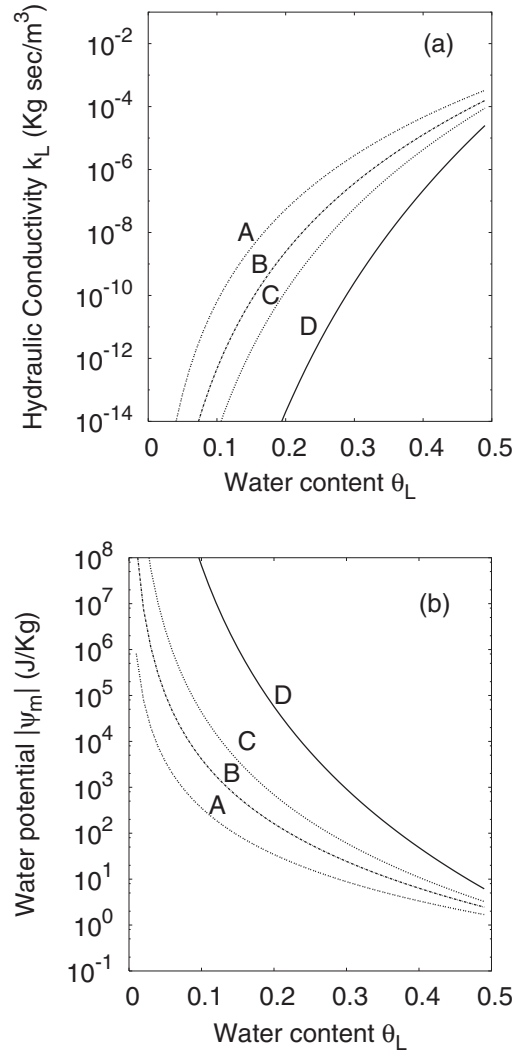

FIG. 2. Dependence of (a) $k_{L}$ and (b) $\left|\psi_{m}\right|$ on $d_{g}$. (A) $d_{g}$ $=0.100 \mathrm{~mm}$, (B) $d_{g}=0.050 \mathrm{~mm}$, (C) $d_{g}=0.030 \mathrm{~mm}$, and (D) $d_{g}$ $=0.010 \mathrm{~mm}$.

$$
\begin{aligned}
& k_{L}(\mathbf{r}, t)=k_{L 0}\left(\frac{\theta_{L}(\mathbf{r}, t)}{\theta_{S}}\right)^{2 b+3}, \\
& \psi_{m}(\mathbf{r}, t)=\psi_{m 0}\left(\frac{\theta_{L}(\mathbf{r}, t)}{\theta_{S}}\right)^{-b},
\end{aligned}
$$

where

$$
\begin{gathered}
\psi_{m 0}=-0.5 d_{g}^{-1 / 2}, \\
k_{L 0}=1.0 \times 10^{-3} \psi_{m 0}^{-2}, \\
b=-2 \psi_{m 0}+0.2 \sigma_{g} .
\end{gathered}
$$

$\psi_{m 0}$ and $k_{L 0}$ are material constants. The units of $\psi_{m 0}$ and $k_{L 0}$ are $\mathrm{J} / \mathrm{kg}$ and $\mathrm{kg} \mathrm{s} / \mathrm{m}^{3}$, respectively. $d_{g}$ and $\sigma_{g}$ are the geometric mean diameter and standard deviation of the particles, respectively (the unit is $\mathrm{mm}$ ). The index $b$ is a phenomenological parameter for soil-water systems. The relations (13) and (14) are explained by some naive theories assuming the power-law distribution of pore size [37]. The dependences of $k_{L}(\mathbf{r}, t)$ and $\left|\psi_{m}(\mathbf{r}, t)\right|$ on the diameter $d_{g}$ are indicated in Fig. 2. Further experimental examination may be required to confirm the validity for starch-water mixtures. We, however, expect that the important feature of this model is the functional shape of the two relations $k_{L}\left(\theta_{L}\right)$ and $\psi_{m}\left(\theta_{L}\right)$ and that the following results are robust against tiny modifications.

The flux of vapor is described by Fick's law

$$
J_{V}(\mathbf{r}, t)=-D_{V} \epsilon_{V} \theta_{G}(\mathbf{r}, t) \nabla \rho_{V}(\mathbf{r}, t),
$$

where $D_{V}$ is the diffusion constant of vapor and $\epsilon_{V}$ is the tortuosity factor. The density of vapor is given by

$$
\rho_{V}(\mathbf{r}, t)=\rho_{V 0} h(\mathbf{r}, t),
$$

where $\rho_{V 0}$ is the saturation vapor density and the relative humidity $h(\mathbf{r}, t)$ is related to the matric potential by Eq. (8).

The evolution equation of $\psi_{m}(\mathbf{r}, t)$ is obtained from Eqs. (8)-(19):

$$
f\left(\psi_{m}\right) \frac{\partial \psi_{m}(\mathbf{r}, t)}{\partial t}=\nabla\left[k_{m}(\mathbf{r}, t) \nabla \psi_{m}(\mathbf{r}, t)\right]
$$

where

$$
\begin{gathered}
f\left(\psi_{m}\right)=\frac{\left[\rho_{L}-\rho_{V}(\mathbf{r}, t)\right] \theta_{S}}{-b \psi_{m}(\mathbf{r}, t)}\left(\frac{\psi_{m 0}}{\psi_{m}(\mathbf{r}, t)}\right)^{1 / b}+\frac{M_{w} \rho_{V}(\mathbf{r}, t) \theta_{G}(\mathbf{r}, t)}{R T} \\
k_{m}(\mathbf{r}, t)=k_{L}(\mathbf{r}, t)+k_{V}(\mathbf{r}, t) \\
k_{V}(\mathbf{r}, t)=\frac{D_{V} \epsilon_{V} \theta_{G}(\mathbf{r}, t) \rho_{V}(\mathbf{r}, t) M_{w}}{R T}
\end{gathered}
$$

The boundary condition is that the evaporation occurs only at the top surface

$$
\begin{gathered}
-\left.\left(k_{V}+k_{L}\right) \frac{\partial \psi_{m}}{\partial z}\right|_{\text {top }}=-E_{p} \frac{h(z=0, t)-h_{a}}{1-h_{a}}, \\
\left.\frac{\partial \psi_{m}}{\partial z}\right|_{\text {bottom }}=0 \\
\left.\mathbf{n} \cdot \nabla \psi_{m}\right|_{\text {side }}=0
\end{gathered}
$$

where $E_{p}$ is the evaporation rate for the state that vapor is saturated on the top surface and $h_{a}$ is the atmospheric humidity and $\mathbf{n}$ is the normal vector at the side boundary. Note that the relative humidity at the top surface $h(z=0, t)$ changes, so the evaporation rate is not constant [42].

\section{Combination of two fields}

The volume shrinkage occurs due to drying, which is the effect from the water content field to the stress field. This effect is incorporated into the model such that the natural length of each spring is an increasing function of the water content $\theta_{L}(\mathbf{r}, t)$, i.e., the natural length $a_{\alpha \beta}$ of the spring between the lattice points $\alpha$ and $\beta$ is determined as

$$
\begin{gathered}
a_{\alpha \beta}=a_{0 \alpha \beta}\left\{1+\kappa\left(\frac{\theta_{L \alpha}+\theta_{L \beta}}{2}-\theta_{S}\right)\right\}, \\
\theta_{L \alpha}=\theta_{L}\left(\frac{L_{x}}{N_{x}} X, \frac{L_{y}}{N_{y}} Y, \frac{L_{z}}{N_{z}} Z, t\right),
\end{gathered}
$$




$$
a_{0 \alpha \beta}= \begin{cases}a_{0} & (\mathrm{NN}), \\ \sqrt{2} a_{0} & (\mathrm{NNN}) .\end{cases}
$$

The parameters of the stress field, $k_{1}, k_{2}$, the expansion coefficient $\kappa$, and the critical force for breaking $F_{c}$, are supposed to be constant for simplicity. The changes in the stress field may affect the water content field (or the water potential), however, this effect is assumed to be negligible.

We also assume that evaporation does not occur from the crack surfaces as well as the side boundaries. Therefore, in our model, all the field variables, such as the water content field $\theta_{L}(\mathbf{r}, t)$ and $\psi_{m}(\mathbf{r}, t)$ are the function of the depth $z$ and time $t$. The argument of the field variables is changed from $(\mathbf{r}, t)$ to $(z, t)$ hereafter. The drainage effect, i.e., the role of the evaporation through the crack, will be discussed in Sec. IV.

\section{Time evolution}

The initial states in our simulations are prepared so that the pores are saturated,

$$
\theta_{L}(z, t=0)=\theta_{S},
$$

and randomly chosen $1 \%$ of the springs at the surface $(z$ $=0)$ are broken. We repeated the following procedures at each time step.

(1) Time steps by $d t$ and the water potential $\psi_{m}(z, t)$ are calculated from Eq. (20) and the water content is obtained from Eq. (14).

(2) The natural length of each spring is determined from the water content field $\theta_{L}(z, t)$ by Eqs. (27)-(29).

(3) The equilibrium configuration of the stress field is calculated so that the elastic energy (6) has the minimal value by the conjugate gradient method [43] with a tolerance $10^{-4}$.

(4) If the force given on a spring exceeds the critical value $F_{c}$, it breaks, i.e., the corresponding spring constant changes to zero. If more than one spring has a load larger than $F_{c}$, we break the one which has the maximum force.

(5) The procedures 3 and 4 are repeated until no spring is broken, and then returned to the first procedure.

\section{NUMERICAL RESULTS}

\section{A. Typical patterns}

The parameters used in the simulations are displayed in Table I. The elastic and fracture parameters $k_{1}, k_{2}$, and $F_{c}$ are chosen as it works. Below we show the results for the particle diameter $d_{g}=0.050 \mathrm{~mm}$ except for Figs. 4 and 13. The typical results at $t=100 \mathrm{~h}$ which corresponds to the middle of the stage 3 are shown in Fig. 3. The water content decreases due to drying and a region where the water content field changes sharply exists, i.e., the drying front exists near $z$ $\sim 0.4 \mathrm{~cm}$. The nonuniform contraction near the drying front consequently increases the stress in this region and fractures occur. In the front of cracks, the stress intensifies and the tensile springs [denoted by red or dark gray in Fig. 3(b)] accumulate. Compressive springs (blue or light gray) accumulate between the crack tips. Figure 3(c) represents the
TABLE I. Parameters of the water content field [37] and the stress field.

\begin{tabular}{ll}
\hline system size $L_{x} \times L_{y} \times L_{z}$ & $2.5 \times 2.5$ \\
$N_{x} \times N_{y} \times N_{z}$ & $\times 2.5 \mathrm{~cm}^{3}$ \\
density of liquid water $\rho_{L}$ & $50 \times 50 \times 50$ \\
density of saturation vapor $\rho_{V 0}$ & $1.0 \times 10^{3} \mathrm{~kg} \mathrm{~m}^{-3}$ \\
saturation water content $\theta_{S}$ & $1.7 \times 10^{-2} \mathrm{~kg} \mathrm{~m}^{-3}$ \\
mass of a mol of water $M_{w}$ & 0.5 \\
gas constant $R$ & $18 \mathrm{~g} \mathrm{~mol}^{-1}$ \\
temperature $T$ & $8.3 \mathrm{~J} \mathrm{~mol}^{-1} \mathrm{~K}^{-1}$ \\
$R T / M_{w}$ & $293 \mathrm{~K}^{5}$ \\
diffusion constant of vapor $D_{V}$ & $1.35 \times 10^{5} \mathrm{~J} \mathrm{~kg}^{-1}$ \\
tortuosity factor $\epsilon_{V}$ & $2.5 \times 10^{-5} \mathrm{~m}^{2} \mathrm{~s}^{-1}$ \\
evaporation rate $E_{p}$ & 0.66 \\
atmospheric humidity $h_{a}$ & $0.01 \mathrm{~g} \mathrm{~cm}^{-2} \mathrm{~h}^{-1}$ \\
geometric mean particle diameter $d_{g}$ & 0.5 \\
geometric standard deviation & $0.01 \sim 0.10 \mathrm{~mm}$ \\
of a particle diameter $\sigma_{g}$ & 1.0 \\
spring constants $k_{1} a_{0}, k_{2} a_{0}$ & \\
critical force for breaking $F_{c}$ & $1.0,0.5$ \\
expansion coefficient $\kappa$ & 0.003 \\
time step $d t$ & 0.2 \\
\hline \hline
\end{tabular}

springs broken between $t=90$ and $100 \mathrm{~h}$. The region where fractures occur actively forms a polygonal pattern and the trace of such a region develops into the prismatic structure of the cracks. These results well capture the features observed in the real experiments $[13,15-18]$. Next, we focus on the spatiotemporal behavior of the key elements, i.e., the water content field, the stress field, and the crack pattern.

\section{B. Water content field}

The time evolution of the normalized total mass of liquid water $\int \theta_{L}(z, t) / \theta_{S} d z$ is displayed in Fig. 4. The two different stages are observed as in the experiments [13], i.e., constant slope stage and slower drying stage. The former and the latter correspond to the stages 1 and 3, respectively, and the stage 2 connects them. The time when the stage 1 switches to the stage 3 depends on the particle diameter $d_{g}$. From the Kelvin equation (8) and the relation between the water content and the water potential, Eq. (14) (shown in Fig. 2), the larger the particle diameter, the larger the amount of water that evaporates.

The depth dependences of the several functions related to the water content field are depicted in Figs. 5-8. In the stage $1(0<t<50 \mathrm{~h})$, the volumetric water content $\theta_{L}$ decreases uniformly and the flux of liquid water dominates the transport of water. In the stage $3(t>80 \mathrm{~h})$, the drying front appears and propagates inward. The dominant process of the transportation of water differs between above and below the front, i.e., the flux of liquid water $J_{L}$ dominates below the drying front while the flux of vapor $J_{V}$ replaces it (Figs. 5 and 6). 
(a)

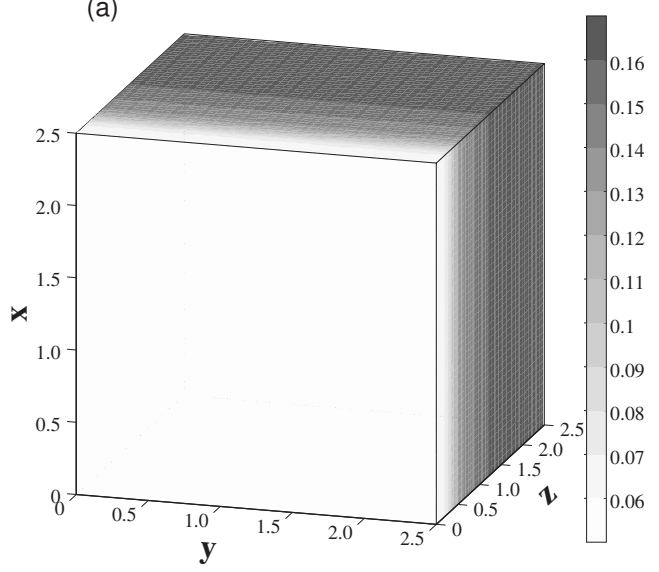

(b)

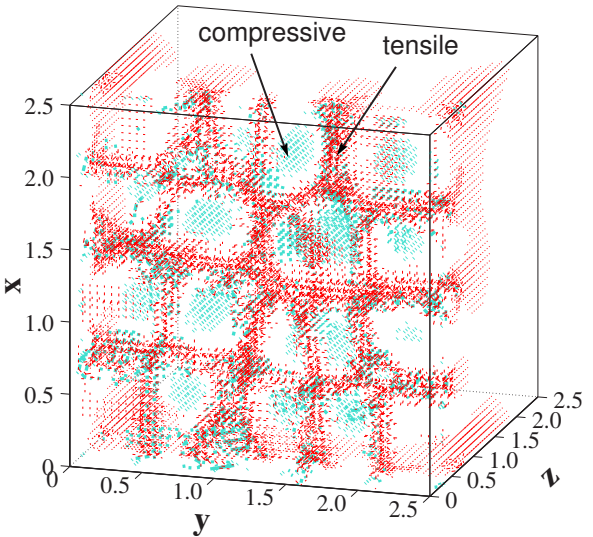

(c)

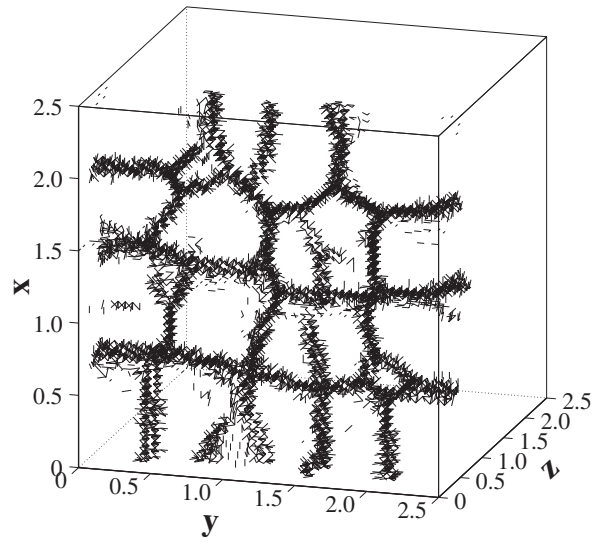

FIG. 3. (Color online) Typical results of the numerical simulations. The unit of axis is $\mathrm{cm}$. (a) The water content field $\theta_{L}$ at $t=100 \mathrm{~h}$. (b) The stress field at $t=100 \mathrm{~h}$. The springs of which force is more than $0.4 F_{c}$ are plotted in red (tensile) and blue (compressive), or for the grayscale image, in dark gray (tensile) and light gray (compressive). In the front of cracks, the stress intensifies and the tensile springs accumulate. Compressive springs accumulate between the crack tips. (c) The crack pattern. The springs broken in $90<t<100 \mathrm{~h}$ are shown.

Above the drying front, the absolute value of the water potential $\left|\psi_{m}(z, t)\right|$ increases sharply to the order of $R T / M_{w}\left(\sim 10^{5} \mathrm{~J} / \mathrm{kg}\right)$ and the relative humidity decreases significantly below 1 (Figs. 7 and 8). The contribution of the capillary water between micrometer-size particles to the water potential is on the order of $10^{2} \mathrm{~J} / \mathrm{kg}$. So, the effects of the

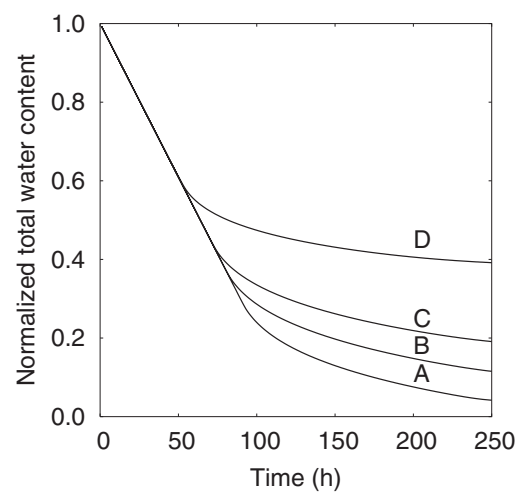

FIG. 4. Evolution of normalized total mass of liquid water $\int \theta_{L}(z, t) / \theta_{S} d z$. (A) $d_{g}=0.100 \mathrm{~mm}$, (B) $d_{g}=0.050 \mathrm{~mm}$, (C) $d_{g}$ $=0.030 \mathrm{~mm}$, and (D) $d_{g}=0.010 \mathrm{~mm}$. electrical and van der Waals force in the vicinity of the solidliquid interface are supposed to be important [38].

\section{Stress field}

Figure 9 displays the water content field, the normalized elastic energy density, and the stress field on the vertical section of the system at $t=120 \mathrm{~h}$. The energy density is nor-

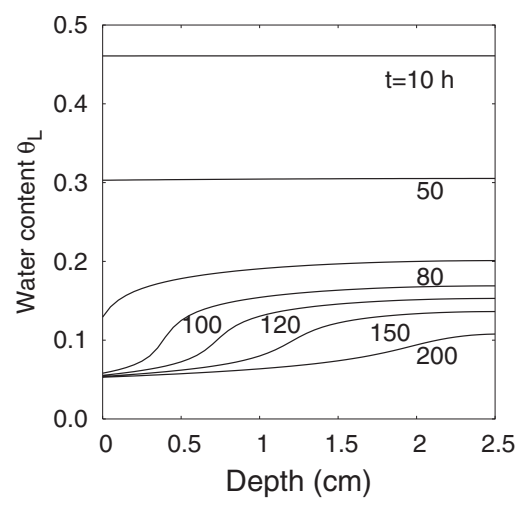

FIG. 5. Volumetric water content $\theta_{L}$ vs depth $z$. 


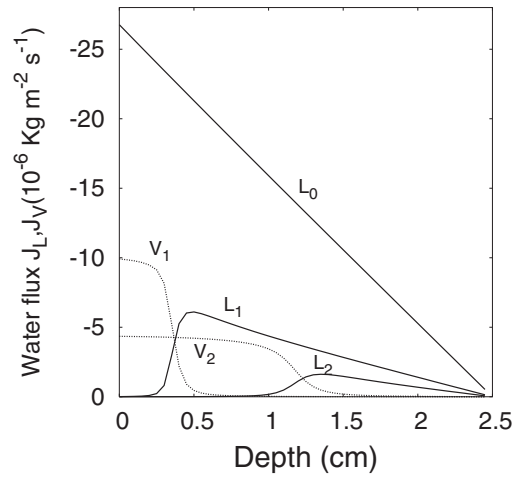

FIG. 6. Flux of liquid water $J_{L}$ and flux of vapor $J_{V}$ vs depth $z$. $L_{0}: \quad J_{L}(t=50 \mathrm{~h}), \quad L_{1}: J_{L}(t=100 \mathrm{~h}), \quad V_{1}: J_{V}(t=100 \mathrm{~h}), \quad L_{2}: J_{L}(t$ $=150 \mathrm{~h})$, and $V_{2}: J_{V}(t=150 \mathrm{~h}) . J_{V}(t=50 \mathrm{~h})$ is on the order of $10^{-11} \mathrm{Kg} \mathrm{m}^{-2} \mathrm{~s}^{-1}$.

malized by the maximum value in the vertical section. The drying front where the water content sharply changes and the region where the tip of cracks exists and the region where the tensile springs [denoted by red or dark gray in Fig. 9(c)] accumulate, all coincide. Compressive springs (blue or light gray) accumulate between the crack tips and stress does not concentrate in the region far from the drying front. The trace of the fractured region forms the columnar crack pattern eventually observed. There is a crack whose tip does not follow the drying front (at $x \sim 2.0 \mathrm{~cm}$ ). This is an elementary column merger event.

\section{Cracks}

Cracks are formed when the uniformity of the water content distribution is lost and the tips of cracks propagate inward. The time evolution of $\sum_{\tau<t} l(z, \tau)$ is shown in Fig. 10, where $l(z, \tau)$ represents the number of springs broken at $(z, \tau) . \Sigma_{\tau<t} l(z, \tau)$ corresponds to the cumulative crack length at $(z, t)$. The area surrounded by the two curves at different times corresponds to the number of springs broken between those times. From the fact that the difference between the distribution of broken springs for two adjacent times has finite support, the active cracking area is localized in the $z$ direction. The relation between the position of active crack-

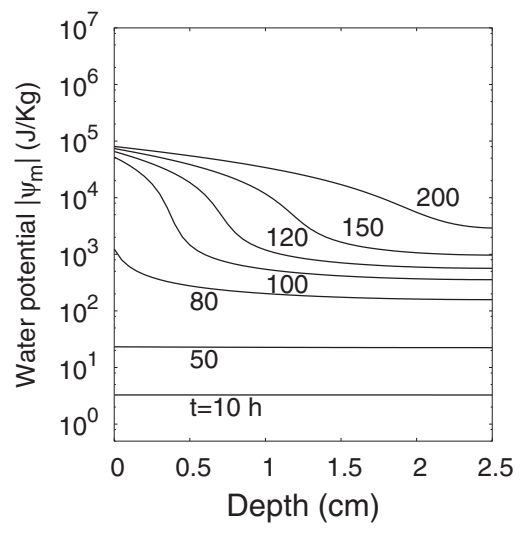

FIG. 7. Water potential $\left|\psi_{m}\right|$ vs depth $z$.

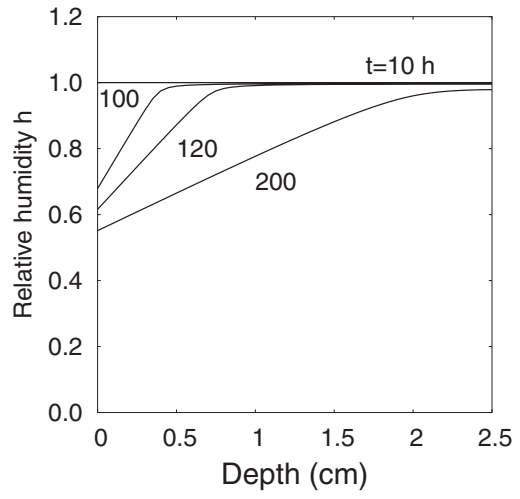

FIG. 8. Relative humidity $h$ vs depth $z$.

ing area and the drying front can be checked as follows. The space-time plots of $d \theta_{L}(z, t) / d z, \Sigma_{X, Y} E_{\alpha}$ and $l(z, t)$, are shown as gray scale images in Fig. 11. The energy density is normalized by the maximum value in all region. It is confirmed that the active cracking area moves inward following the drying front. However, the number of the broken springs is not the simple function of the water content $\theta_{L}(z, t)$ or its derivative.

The typical patterns of cracks formed up to the final state $(t=200 \mathrm{~h})$ are shown in Fig. 12. The polygonal columnar structure can be seen despite of the anisotropy of the cubic lattice. In Ref. [18], two mechanisms for changing scale are suggested, i.e., merging and column initiation from a vertex. In Fig. 12, both processes are observed. These features well capture the experiments of starch-water mixtures [13,15-18]. Now, we analyze the depth dependence of the final crack pattern. Figure 13 exhibits the depth dependence of the cumulative crack length up to the final state with double logarithmic plot to compare with the experimental data. In contrast to the real experiments, we can easily change the physical parameters. If we change the particle diameter $d_{g}$, it affects the $\psi_{m 0}$ through phenomenological Eq. (15). The size of columns decreases as the particle diameter increases. Though it is difficult to state definitely, there is a regime in which the number of broken springs decreases with a power law with exponent between -0.5 and -2 .

\section{DISCUSSION}

In this section, we note several points such as the translation of the evolution equation from the matric potential $\psi_{m}(z, t)$ to the water content $\theta_{L}(z, t)$, the drainage effect of cracks, the geological columnar joints, the feedback from the stress field to the water content field, the characterization of the crack patterns, and the properties of the granule-water mixtures.

First, we discuss the drying front which is obtained from the evolution equation of the water potential [Eq. (20)]. Changing the variable from $\psi_{m}(z, t)$ to $\theta_{L}(z, t)$ by Eq. (14), we get

$$
\rho_{L}\left\{1+g\left(\theta_{L}\right)\right\} \frac{\partial \theta_{L}(z, t)}{\partial t}=\frac{d}{d z}\left(D_{L}(z, t) \frac{d}{d z} \theta_{L}(z, t)\right),
$$


(a)

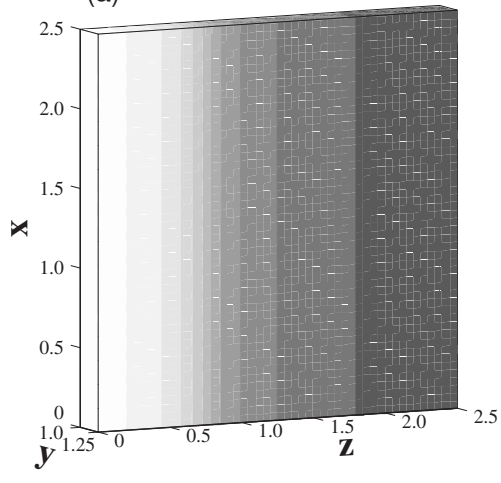

(b)

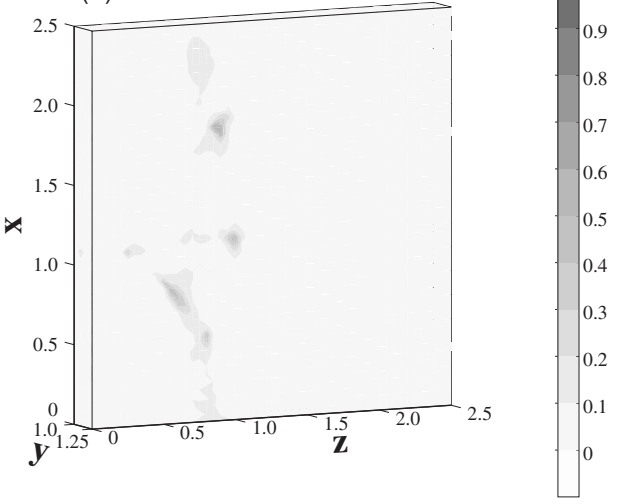

(c)

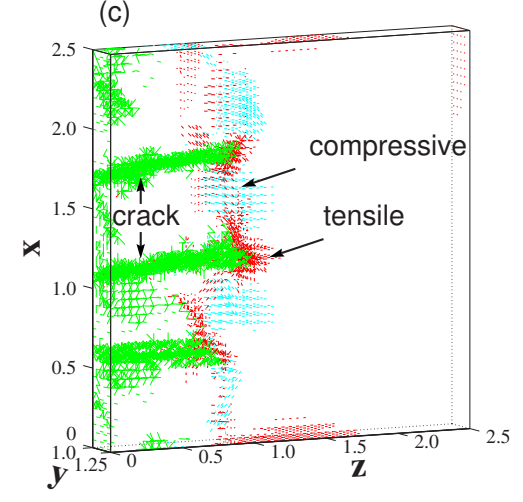

FIG. 9. (Color online) The unit of axis is $\mathrm{cm}$. (a) The water content field, (b) the normalized elastic energy density, and (c) the stress field are displayed in $1.0<y<1.25 \mathrm{~cm}$ at $t=120 \mathrm{~h}$. The springs of which force is more than $0.3 F_{c}$ are plotted in red (tensile) and blue (compressive), or for the grayscale image, in dark gray (tensile) and light gray (compressive). The broken springs are plotted in green, or for grayscale image, in light gray. In the drying front where the water content sharply changes, the tip of cracks exists and the tensile springs accumulate. Compressive springs accumulate between the crack tips.

where

$$
\begin{gathered}
g\left(\theta_{L}\right)=-\frac{\rho_{V}(z, t)}{\rho_{L}}\left(1+\frac{b \psi_{m}(z, t) M_{w} \theta_{G}(z, t)}{R T \theta_{L}(z, t)}\right), \\
D_{L}(z, t)=\frac{-b \psi_{m}(z, t)\left[k_{L}(z, t)+k_{V}(z, t)\right]}{\theta_{L}(z, t)} .
\end{gathered}
$$

$g\left(\theta_{L}\right)$ is of order $10^{-4}$ due to $\rho_{V} \ll \rho_{L}$; therefore, if we ignore $g\left(\theta_{L}\right)$, the time evolution of $\theta_{L}$ can be considered to obey the nonlinear diffusion equation with variable diffusion coefficient $D_{L}(z, t)$. In our simulations the diffusion coefficient $D_{L}(z, t)$ has a minimum near the drying front (Fig. 14) as Goehring et al. [18] pointed out in their experiments. The nonlinearity of the diffusion coefficient is crucial for the water content distribution which has a concave regime.

Second, we discuss the assumption that cracks do not affect the water content field. Assuming the crack aperture width $l_{c} \sim 10^{-1} \mathrm{~mm}$ and the propagation speed of the drying front $v_{w} \sim 10^{-5} \mathrm{~mm} / \mathrm{s}$ and the volumetric gas content near the drying front $\theta_{G} \sim 0.4$, the length of the vapor diffusion in $\tau_{w}=l_{c} / v_{w}$ is on the order of $\sqrt{D_{v} \epsilon_{V} \theta_{G} \tau_{w}} \sim 10^{2} \mathrm{~mm}$. Because this is much larger than the crack aperture width, the density of vapor in a crack aperture is determined quasistatically by the water potential on the crack surface. Therefore, the effect of cracks on the dynamics of the water content field as the

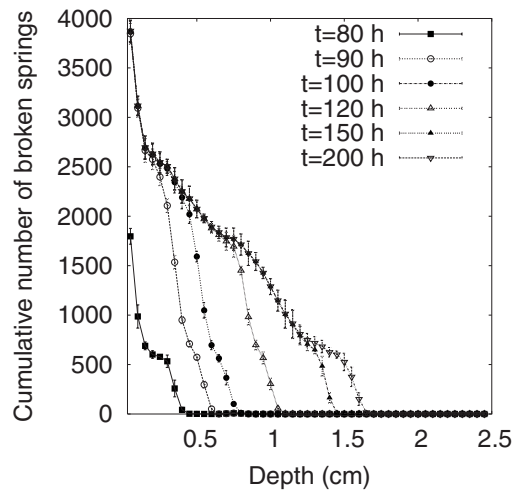

FIG. 10. Time evolution of the cumulative number of the broken springs at each horizontal cross section. The results obtained from seven different initial conditions are averaged at a given depth $z$. 

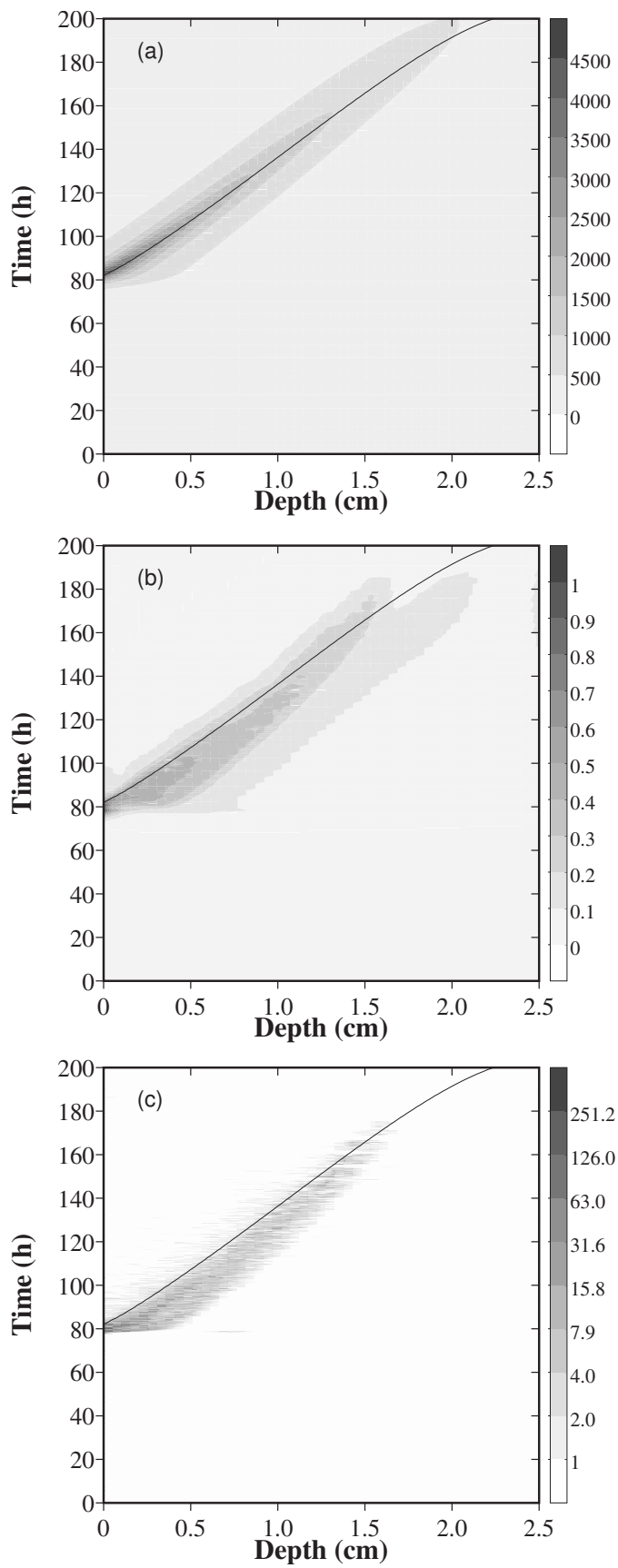

FIG. 11. Space-time plot of (a) $d \theta_{L} / d z$, (b) the normalized elastic energy density, and (c) the number of the broken springs. These fields are averaged over the cross section at a given $z$. The line indicates the depth where the flux of vapor is equal to that of liquid water (see Fig. 6), which corresponds to the position of the drying front.

boundary condition can be negligible above the drying front because the flux of vapor is dominant in this region. The flux of liquid water may be affected by the cracks in the region where the two fluxes are comparable. However, we infer that the flux of water does not change significantly because the region is limited in the vicinities of the crack tips. Hence the assumption is valid except for a small effect near the drying front.
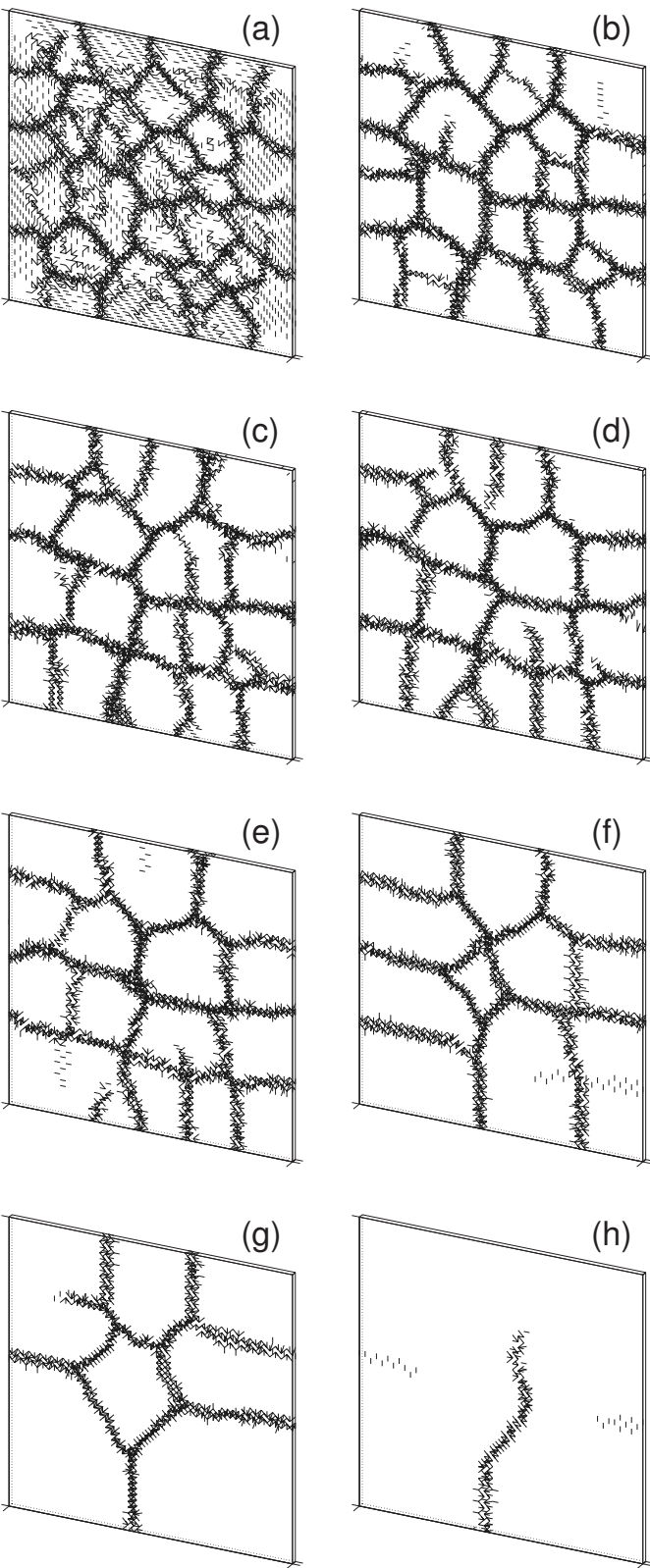

FIG. 12. Cross sections of the pattern of cracks at $t=200 \mathrm{~h}$. (a) $z=0 \mathrm{~cm}$, (b) $z=0.15 \mathrm{~cm}$, (c) $z=0.3 \mathrm{~cm}$, (d) $z=0.45 \mathrm{~cm}$, (e) $z$ $=0.6 \mathrm{~cm}$, (f) $z=0.9 \mathrm{~cm},(\mathrm{~g}) z=1.2 \mathrm{~cm}$, and (h) $z=1.5 \mathrm{~cm}$.

For studying the effect of cracks on the water content field, we also perform numerical simulations based on the different assumptions that the transportation of liquid water obeys the linear diffusion equation and the diffusion constant in cracks is larger than in medium. The result, however, is that the broken springs do not form planes to become crack surfaces but form some clusters and no stable prismatic pattern is observed. Recently, Sørenssen et al. [25] report a similar fracture simulation under the condition that the quantity which determines the local volume reduction diffuses to the boundary of the solid and find that the zone where the fracture occurs actively propagates and the fracture patterns resemble a forest of trees, i.e., there are many branches of cracks. In order to obtain the prismatic fracture pattern under 


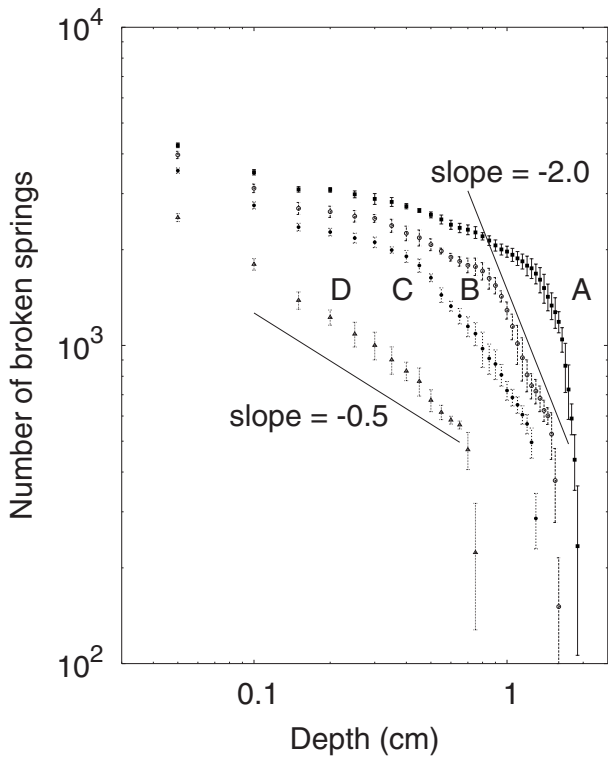

FIG. 13. The cumulative number of the broken springs at each cross section at the final state $(t=200 \mathrm{~h})$ for various particle diameters, (A) $d_{g}=0.100 \mathrm{~mm},\left(\right.$ B) $d_{g}=0.050 \mathrm{~mm}$, (C) $d_{g}=0.030 \mathrm{~mm}$, and (D) $d_{g}=0.010 \mathrm{~mm}$. The results obtained from seven different initial conditions are averaged for each particle diameter.

the condition that the water content field does not affect the stress field except for determining the volume shrinkage rate, the mechanism that the external field forms the front by itself without cracks would be necessary. It is the nonlinear diffusion of water in our simulations.

Third, let us consider the other columnar joint formation phenomenon, i.e., the geological columnar joint formation driven by lava cooling process. It is accepted that the cooling process of lava is the combination of the linear diffusion the of heat and the convective heat loss through cracks $[6,7,9,26,27]$. On the other hand, in our spring network model we obtained the prismatic structure of cracks in the condition that cracks does not affect the water content field and the water pressure do not affect the stress field. There is the difference in the external field, i.e., the thermal field above the front is nearly constant [26] and the water content field below the front is nearly constant $[17,18]$. So it is a future work to study numerically the cooling joints with taking account of the convective heat loss through cracks and the external stresses, i.e., the horizontal tectonic stress, the vertical overburden stress, and the local pore pressure.

Fourth, we discuss the feedback from the stress field to the water content field. We ignore this feedback, i.e., the water potential is assumed not to be affected by the change of the stress field. In our simulations, as described in Sec. III $\mathrm{B}$, the contribution of the capillary water pressure to the water potential becomes much smaller than the absolute value when the water content decreases. Therefore, we infer that, when the drying front appears, the deformation does not change significantly the water potential through the water pressure. This inference is consistent with our assumption. However, it is possible that the deformation may affect the properties of water on the particle surfaces and the shapes of

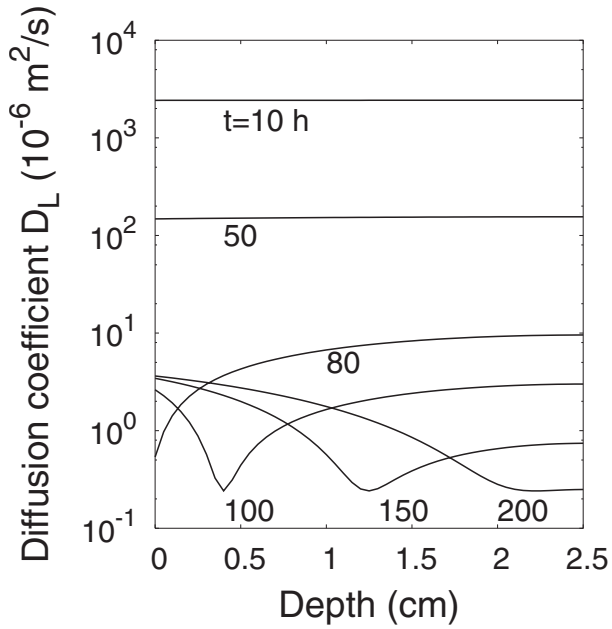

FIG. 14. Diffusion coefficient $D_{L}$ defined by Eq. (33).

the water-vapor interfaces. It is a future subject to investigate the contribution of these effects to the water potential.

Fifth, we discuss the characterization of the crack patterns. The fracture occurs near the propagating front of the water content field. The trace of the fractured region forms the columnar pattern with polygonal cross sections. The size of columns increases with depth. In this paper, as a key quantity, we focus on the crack length (i.e., the number of broken springs) which is supposed to be proportional to the surface energy in connection with the Griffith theory [44]. Other characterizations, such as the mean polygon area and number of polygon sides, seem to be worth analyzing as a future work. However, they should require larger system size and careful definition of the quantities. If the pattern at the cross section is assumed to be isotropic, the characteristic length of the pattern is inversely proportional to the crack length, so it is possible to compare the depth dependence of the cell area at the cross section with the experimental results. We suggest that the drying granule-water mixtures of larger particles forms the smaller size of columns. the dependence of the size of columns on the particle diameter is a future subject.

Finally, we discuss the properties of the granule-water mixtures. We obtain the prismatic pattern of cracks by employing the relations for general soils in our simulations. For the present, it is known that only the combination of starch and water forms the prismatic fracture pattern among drying mixtures of various combinations of granules and liquids. For understanding why dried mixtures of other granules and water do not form the columnar structure, it is necessary to measure experimentally various material properties such as the expansion coefficient and the dependences of the water potential and the hydraulic conductivity on the water concentration.

\section{CONCLUSIONS}

We study the drying process and the columnar fracture process in granule-water mixtures. The mixture is assumed to be the elastic porous medium and each process is repre- 
sented by a phenomenological model with the water potential and a spring network, respectively. We assume that the cracks do not affect the water content field as a boundary condition and ignore the feedback from the stress field to the water content field. In the numerical simulations, we find that the water content distribution has a propagating front and the pattern of cracks exhibits a prismatic structure.

\section{ACKNOWLEDGMENTS}

We acknowledge Y. Sasaki, I. Aoki, Y. Kuramoto, S. Shinomoto, H. Nakao, and all members of the nonlinear dynamics group, Kyoto University, for fruitful discussions and encouragements. The numerical calculations were carried out on Altix3700 BX2 at YITP in Kyoto University.
[1] J. Walker, Sci. Am. 255, 178 (1986).

[2] S. Folley, Philos. Trans. R. Soc. London, Ser. A 212, 170 (1694).

[3] M. Mallet, Philos. Mag. 50, 122 (1875).

[4] D. L. Peck and T. Minakami, Geol. Soc. Am. Bull. 79, 1151 (1968).

[5] A. Aydin and J. M. DeGraff, Science 239, 471 (1988).

[6] M. Reiter, M. W. Barroll, J. Minier, and G. Clarkson, Tectonophysics 12, 241 (1987).

[7] J. M. Degraff and A. Aydin, J. Geophys. Res. 98, 6411 (1993).

[8] K. A. Grossenbacher and S. M. McDuffie, J. Volcanol. Geotherm. Res. 69, 95 (1995).

[9] P. Budkewitsch and P.-Y. Robin, J. Volcanol. Geotherm. Res. 59, 219 (1994).

[10] E. A. Jagla and A. G. Rojo, Phys. Rev. E 65, 026203 (2002).

[11] R. Saliba and E. A. Jagla, J. Geophys. Res. 108, 2476 (2003).

[12] G. Müller, J. Volcanol. Geotherm. Res. 86, 93 (1998).

[13] G. Müller, J. Geophys. Res. 103, 15239 (1998).

[14] T. Mizuguchi, A. Nishimoto, S. Kitsunezaki, Y. Yamazaki, and I. Aoki, in Powders and Grains 2001, edited by Y. Kishino (Balkema, Lisse, 2001), p. 55.

[15] A. Toramaru and T. Matsumoto, J. Geophys. Res. 109, B02205 (2004).

[16] L. Goehring and S. W. Morris, Europhys. Lett. 69, 739 (2005).

[17] T. Mizuguchi, A. Nishimoto, S. Kitsunezaki, Y. Yamazaki, and I. Aoki, Phys. Rev. E 71, 056122 (2005).

[18] L. Goehring, S. W. Morris, and Z. Lin, Phys. Rev. E 74, 036115 (2006).

[19] A. Groisman and E. Kaplan, Europhys. Lett. 25, 415 (1994).

[20] S. Kitsunezaki, Phys. Rev. E 60, 6449 (1999).

[21] The names "primary" and "secondary" cracks seem to imply the temporal order of the phenomena. In some cases, however, only the "secondary" crack forms under specific conditions. Therefore, more neutral names such as "type I" and "type II" [17] may be adequate.

[22] Y. Hayakawa, Phys. Rev. E 50, R1748 (1994).

[23] B. I. Yakobson, Phys. Rev. Lett. 67, 1590 (1991).
[24] T. Boeck, H. A. Bahr, S. Lampenscherf, and U. Bahr, Phys. Rev. E 59, 1408 (1999).

[25] A. Malthe-Sørenssen, B. Jamtveit, and P. Meakin, Phys. Rev. Lett. 96, 245501 (2006).

[26] H. C. Hardee, J. Volcanol. Geotherm. Res. 7, 211 (1980).

[27] J. M. DeGraff, P. E. Long, and A. Aydin, J. Volcanol. Geotherm. Res. 38, 309 (1989).

[28] C. Allain and L. Limat, Phys. Rev. Lett. 74, 2981 (1995).

[29] A. Yuse and M. Sano, Nature (London) 362, 329 (1993).

[30] O. Ronsin and B. Perrin, Europhys. Lett. 38, 435 (1997).

[31] O. Ronsin and B. Perrin, Phys. Rev. E 58, 7878 (1998).

[32] T. Komatsu and S. Sasa, Jpn. J. Appl. Phys. 36, 391 (1997).

[33] E. R. Dufresne, E. I. Corwin, N. A. Greenblatt, J. Ashmore, D. Y. Wang, A. D. Dinsmore, J. X. Cheng, X. S. Xie, J. W. Hutchinson, and D. A. Weitz, Phys. Rev. Lett. 91, 224501 (2003).

[34] E. R. Dufresne, D. J. Stark, N. A. Greenblatt, J. X. Cheng, J. W. Hutchinson, L. Mahadevan, and D. A. Weitz, Langmuir 22, 7144 (2006).

[35] N. Lu and W. J. Likos, Unsaturated Soil Mechanics (John Wiley \& Sons, Hoboken, NJ, 2004).

[36] The water potential has several aliases such as suction, headand soil-water potential.

[37] G. S. Campbell, Soil Physics with BASIC-Transport Models for Soil-Plant Systems (Elsevier, Amsterdam, 1985).

[38] S. Iwata, T. Tabuchi, and B. P. Warkentin, Soil-Water Interactions (Marcel Dekker, New York, 1988).

[39] T. Mizuguchi, A. Nishimoto, S. Kitsunezaki, Y. Yamazaki, and I. Aoki (unpublished).

[40] T. S. Komatsu, J. Appl. Meteorol. 42, 1330 (2003).

[41] T. S. Komatsu, J. Phys. Soc. Jpn. 70, 3755 (2001).

[42] In order to simulate the constant evaporation rate devised in Ref. [18], feedback control is required.

[43] W. H. Press, B. P. Flannery, S. A. Teujiksky, and W. T. Etterling, Numerical Recipes (Cambridge University Press, New York, 1988).

[44] A. A. Griffith, Philos. Trans. R. Soc. London, Ser. A 221, 163 (1920). 